\title{
Female, Self-Report
}

National Cancer Institute

\section{Source}

National Cancer Institute. Female, Self-Report. NCI Thesaurus. Code C46108.

An individual who reports to be female, the sex that ordinarily produces ova. 